\title{
Zu von Auer F: Zum Urteil des Europäischen Gerichtshofes vom 9. Dezember 2010 zu einer nationalen Regelung, die die Einfuhr von Blutprodukten verbietet, die nicht aus gänzlich unbezahlt erfolgten Blutspenden stammen. Transfus Med Hemother 2011;38:157-158
}

\author{
Sascha Rolf Lüder \\ DRK-Blutspendedienst West, Hagen, Deutschland
}

Mit Urteil vom 9. Dezember 2010 hat der Europäische Gerichtshof (EuGH) entschieden, dass das europäische Unionsrecht dahin auszulegen ist, dass es einer nationalen Regelung entgegensteht, nach der die Einfuhr von Blut oder Blutbestandteilen aus einem anderen Mitgliedstaat nur unter der auch für inländische Präparate geltenden Bedingung zulässig ist, dass die Spender des Blutes, aus dem diese Präparate gewonnen wurden, nicht nur keine Bezahlung, sondern auch keine Erstattung der Aufwendungen erhalten haben, die ihnen im Rahmen dieser Blutspenden entstanden sind [1]. Das Urteil ist für die Beantwortung der Frage, wann eine als Erstattung von Aufwendungen bezeichnete Geldzahlung zu einem unzulässigen Entgelt für eine Blutspende werden kann, von erheblicher Bedeutung. Die hierauf bezogene Besprechung Friedger von Auers [2] bedarf jedoch der Klarstellung.

Im Kern gilt dies für den Befund, das auf das Blutspendewesen bezogene Recht erachte die Gewährung einer

(...) Aufwandsentschädigung bei der Spendeentnahme auch in Form eines geringfügigen pauschalen Geldbetrages [2]

für zulässig. Dieser Ansatz ist nicht begründet. Weder das staatliche noch das zwischen- oder überstaatliche Recht erteilen den Spendeeinrichtungen eine Befugnis zur Gewährung eines pauschalen Geldbetrages als Aufwandsentschädigung für eine Blutspende.

Zunächst ist festzustellen, was das auf das Blutspendewesen bezogene Recht unter die in $\S 10$ TFG [3] verwendeten
Begriffe der «Unentgeltlichkeit» und der «Aufwandsentschädigung» als solches subsumiert. § 10 TFG sieht vor:

Die Spendeentnahme soll unentgeltlich erfolgen. Der spendenden Person kann eine Aufwandsentschädigung gewährt werden, die sich an dem unmittelbaren Aufwand je nach Spendeart orientieren soll.

Für die Abgrenzung eines unzulässigen Entgeltes zu dem hier entscheidenden Begriff der Aufwandsentschädigung ist neben dem Wortlaut insbesondere der systematische Zusammenhang des $\S 10$ S. 2 TFG sowohl mit S. 1 der Regelung als auch mit den Vorschriften des höherrangigen Rechtes heranzuziehen.

Nach dem Wortlaut des $\S 10$ S. 2 TFG meint «Aufwandsentschädigung» eine Erstattung des vom Spender zur Erbringung seiner Blutspende erbrachten Aufwandes. Dies ist mit der Unentgeltlichkeit einer Leistung vereinbar, da der Ersatz eines bereits erbrachten Aufwandes die Vermögensposition nicht verbessert, sondern nur ausgleicht. Wird der spendenden Person gemäß $\S 10$ S. 2 TFG eine Aufwandsentschädigung gewährt, die sich an dem unmittelbaren Aufwand je nach Spendeart orientieren soll, ist dies damit kein unzulässiges Entgelt, wenn sich die Vermögensposition des Blutspenders hierdurch nicht verbessert.

An dieser Stelle ist die Bestimmung von Art und Umfang der erbrachten Aufwendungen relevant, die mit einer Blutspende verbunden sind. Unstreitig davon erfasst sind diejenigen Aufwendungen, die der Blutspender zur Erbringung seiner Spende tatsächlich auf sich nimmt, insbesondere die auch

\section{KARGER}

Fax +49 7614520714

Information@Karger.de

www.karger.com (c) 2011 S. Karger GmbH, Freiburg

Accessible online at:

www.karger.com/tmh 
in der Gesetzesbegründung zum TFG ausdrücklich genannten Fahrtkosten [4].

Der systematische Zusammenhang zwischen $\S 10$ S. 1 und S. 2 TFG liegt darin, dass S. 2 sich als Ausnahmeregelung gegenüber dem in S. 1 geregelten Gebot der Unentgeltlichkeit der Blutspende darstellt. Dies bedeutet, dass eine Aufwandsentschädigung diesem Gebot nicht zuwiderlaufen darf. Dieser Regelungszusammenhang begrenzt zugleich den möglichen Sinngehalt des $\S 10$ S. 2 TFG. Hiernach darf die Auslegung des Begriffes der «Aufwandsentschädigung» nicht zu einer Umgehung des rechtlichen Gebotes der Unentgeltlichkeit der Blutspende führen.

Von entscheidender Bedeutung ist an dieser Stelle die Auslegung der in $\S 10 \mathrm{~S} .2$ TFG verwendeten Begriffe der «Unentgeltlichkeit» und der «Aufwandsentschädigung» anhand höherrangigen Rechtes. Die Transfusionsrichtlinie 2002/98 [5] der Europäischen Union, die auf die Empfehlung R (95) 14 [6] des Europarates Bezug nimmt, bestätigt den oben angegebenen Befund. So heißt es in der Transfusionsrichtlinie 2002/98/EG in Erwägungsgrund 23:

Freiwillige, unbezahlte Blutspenden sind ein Faktor, der zu hohen Qualitäts- und Sicherheitsstandards für Blut und Blutbestandteile und somit zum Gesundheitsschutz beitragen kann. Die diesbezüglichen Bestrebungen des Europarates sollten unterstützt und alle erforderlichen Maßnahmen ergriffen werden, um freiwillige, unbezahlte Blutspenden durch geeignete Maßnahmen und Initiativen sowie dadurch zu fördern, dass Blutspender größere öffentliche Anerkennung erfahren; damit würde auch die Selbstversorgung der Gemeinschaft verbessert. Die Definition des Europarates für freiwillige, unbezahlte Blutspenden sollte berücksichtigt werden.

Die in Bezug genommene Definition des Europarates in Art. 2 des Anhanges der Empfehlung R (95) 14 lautet wie folgt:

Eine Spende gilt als freiwillig und unentgeltlich, wenn die Person, die Blut, Plasma oder zelluläre Bestandteile spendet, dies aus eigenem, freien Willen tut und keine Bezahlung in Form von Bargeld oder anderen entsprechenden Leistungen erhält. Dies schließt auch eine Vergütung in Form von Freizeit aus, die über den angemessenen Zeitaufwand für die Spende und die An- bzw. Abreise hinausgeht. Geringfügige Anerkennungen, Erfrischungen und die Erstattung der Reisekosten sind mit dem Begriff der freiwilligen, unentgeltlichen Spende vereinbar.

Dies zeigt deutlich, dass die Aufwandsentschädigung bei einer Blutspende mit Ausnahme der Erstattung der Fahrtkosten nicht zu einer Vergütung in Bargeld oder anderen Leistungen führen darf.

Der Umkehrschluss aus $\S 10$ S. 2 TFG legt darüber hinaus nahe, dass auch der Zeitaufwand nur in Form einer Naturalrestitution in Freizeit ersetzt werden dürfe. Über die Zulässigkeit einer finanziellen Vergütung des erbrachten Zeitaufwandes in anderer Form wird keine Aussage getroffen; es erschiene indes als Umgehung des rechtlich formulierten Gebotes der Unentgeltlichkeit der Blutspende, würde man zwar den Spender nicht für das gespendete Blut als materiellen Gegenstand bezahlen, ihm aber die Zeit entgelten, die er für diese Blutspende aufwendet. Denn eine Entschädigung in der Form einer Bezahlung für den Zeitaufwand wäre nichts anderes als eine Entlohnung, zwar nicht für das Blut, wohl aber für die Zeit des Blutspendens. Ein Ersatz der vom Spender aufgewendeten Zeit in Form von Bargeld ist ausgeschlossen [7] (Etwaige steuerrechtliche Aspekte in diesem Zusammenhang bleiben im Rahmen dieses Beitrages unberücksichtigt).

Zusammengefasst bestätigen alle genannten Vorschriften, einschließlich die des zwischen- und überstaatlichen Rechtes, uneingeschränkt das in des $\S 10$ TFG enthaltene Gebot der Unentgeltlichkeit der Blutspende und lassen Aufwandsentschädigungen nur insoweit zu, als dieses Gebot hierdurch nicht umgangen wird.

Was bedeutet dieser Befund nun für die Beantwortung der Frage nach der Befugnis zur Gewährung eines pauschalen Geldbetrages als Aufwandsentschädigung für eine Blutspende?

Der Wortlaut des $\S 10$ S. 2 TFG, der von einer Orientierung der Aufwandsentschädigung am unmittelbaren Aufwand spricht, macht deutlich, dass keine «Spitzabrechnung» des Aufwandes erforderlich ist. Vielmehr ist eine «Orientierung» im Rechtssinne zulässig, damit der Überprüfungsaufwand, zumal bei einem hohen Spendeaufkommen, nicht unverhältnismäßig hoch wird. Hierbei ist der technische Fortschritt insbesondere im Bereich der elektronischen Datenverarbeitung seit Inkrafttreten des TFG zu berücksichtigen.

Andererseits ist zu beachten, dass der Wortlaut des $\S 10 \mathrm{~S}$. 2 TFG eine Orientierung der Aufwandsentschädigung am «unmittelbaren» Aufwand vorschreibt. Auch im Lichte des höherrangigen Rechtes bestätigt dies den Sinn und Zweck des Gebotes der Unentgeltlichkeit der Blutspende. Denn eine Aufwandsentschädigung hat sich deshalb am unmittelbaren Aufwand zu orientieren, damit das Gebot der Unentgeltlichkeit der Blutspende nicht unterlaufen werden kann. Eine Pauschalierung darf nicht dazu führen, dass nicht nur ausnahmsweise, sondern regelmäßig die Zahlung eines über dem Aufwand liegenden Betrages erfolgt, weil dies dem Sinn und Zweck des Gebotes der Unentgeltlichkeit der Blutspende, das vor allem Sicherheitserwägungen dient und einen Beitrag zum Gesundheitsschutz leisten soll, konterkarieren würde.

Diese Auslegung wird unterstützt durch die bereits oben wiedergegebene Begründung, die ausdrücklich davor warnt, dass eine Aufwandsentschädigung nicht den Charakter einer Bezahlung annehmen darf. Damit gilt für die gemäß $§ 10$ S. 2 
TFG vorgesehene Aufwandsentschädigung, dass eine Pauschalierung Grenzen haben muss [8]. Diese liegt im Gebot der Orientierung am unmittelbaren Aufwand im Sinne der Definition des Europarates, deren Bedeutung für die Konturierung des auf die Blutspende bezogenen Rechtes der EuGH in seiner Judikatur jetzt noch einmal bestätigt hat.

Um zu gewährleisten, dass eine «Aufwandsentschädigung» aufgrund einer Pauschalierung nicht zu einem nach § 10 TFG unzulässigen Entgelt wird, ist dafür Sorge zu tragen, dass eine Pauschalierung eine realistische Annäherung an den typischerweise entstehenden tatsächlichen unmittelbaren Aufwand bei einer Blutspende darstellen muss. Denn nur auf diese Weise ist eine «Orientierung» im Rechtssinne gewährleistet; der Spielraum für die Spendeeinrichtungen ist insoweit begrenzt.

Es ist in diesem Zusammenhang hervorzuheben, dass die Bundesrepublik Deutschland bei ihrer Teilnahme an der 30. Internationalen Konferenz des Roten Kreuzes und des Roten Halbmondes im Jahr 2007 mit der Verabschiedung der Entschließung 1 «Gemeinsam für die Menschlichkeit» [9] den Grundsatz der unentgeltlichen Blutspende noch einmal bekräftigt hat. In der Entschließung heißt es wörtlich:

Zugang zu sicherem Blut durch die freiwillige, unentgeltliche Blutspende ist und wird ein wesentliches Ziel der öffentlichen Gesundheit bleiben.

Bezogen auf den Grundsatz der unentgeltlichen Blutspende kann dies nichts anderes bedeuten, als diese Maxime im Sinne des vorgegebenen Rechtsrahmens umzusetzen. Die Gewährung eines pauschalen Geldbetrages als Aufwandsentschädigung für eine Blutspende sieht dieser Rechtsrahmen unabhängig von der jeweiligen Trägerschaft einer Spendeeinrichtung nicht vor.

\section{Literatur}

1 EuGH, Urteil vom 9. Dezember 2010, C-421/09.

2 Von Auer F: Zum Urteil des Europäischen Gerichtshofes vom 9. Dezember 2010 zu einer nationalen Regelung, die die Einfuhr von Blutprodukten verbietet, die nicht aus gänzlich unbezahlt erfolgten Blutspenden stammen. Transfus Med Hemother 2011;38:157-158.

3 Gesetz zur Regelung des Transfusionswesens (Transfusionsgesetz - TFG) vom 1. Juli 1998 (BGBl. I S. 1752) in der Fassung der Bekanntmachung vom 28. August 2007 (BGBl. I S. 2169).

4 BT-Drs. 13/9594 vom 13. Januar 1998, S. 20.

5 Richtlinie 2002/98/EG des Europäischen Parlamentes und des Rates vom 27. Januar 2003 zur Festlegung von Qualitäts- und Sicherheitsstandards für die Gewinnung, Testung, Verarbeitung, Lagerung und Verteilung von menschlichem Blut und Blutbestandteilen und zur Änderung der Richtlinie 2001/83/EG (ABl. EU Nr. L 33 vom 8. Februar 2003).

6 Empfehlung R (95) 14 des Ministerausschusses des Europarates vom 12. Oktober 1995 über den Schutz der Gesundheit von Spendern und Empfängern im Bereich der Bluttransfusion.

7 Deutsch E, Bender A W, Eckstein R, Zimmermann R: Transfusionsrecht, 2. Aufl. Stuttgart, Wissenschaftliche Verlagsgesellschaft, 2007, S. 179 m.w.N

8 Vgl. OLG Hamm, Beschluss vom 8. März 2005, 4 U 19/05.

9 Entschließung 1 der 30. Internationalen Konferenz des Roten Kreuzes und des Roten Halbmondes vom 30. November 2007 